\title{
Crystallography of Some Double Sulfates and Chromates
}

\author{
H. F. McMurdie, ${ }^{*}$ M. C. Morris, ${ }^{*}$ J. deGroot, ${ }^{*}$ and H. E. Swanson** \\ Institute for Basic Standards, National Bureau of Standards, Washington, D.C. 20234
}

(June 2, 1971)

\begin{abstract}
New information is given on cell parameters, density and methods of preparation of 50 compounds of the langbeinite and Tutton salt groups. The langbeinites have the general formula $\left(\mathrm{A}^{+}\right)_{2}\left(\mathrm{~B}^{2+}\right)_{2}\left(\mathrm{XO}_{4}\right)_{3}$ and the Tutton salts the general formula $\left(\mathrm{A}^{+}\right)_{2}\left(\mathrm{~B}^{2+}\right)\left(\mathrm{XO}_{4}\right)_{2} \cdot 6 \mathrm{H}_{2} \mathrm{O}$, where $\mathrm{A}$ is $\mathrm{K}, \mathrm{Rb},\left(\mathrm{NH}_{4}\right)$, $\mathrm{Tl}$ or Cs; $\mathrm{B}$ is $\mathrm{Mg}, \mathrm{Ni}, \mathrm{Cu}, \mathrm{Co}$, or $\mathrm{Zn}$; and $\mathrm{X}$ is $\mathrm{S}$ or Cr. A comprehensive list of references on the crystallography of the compounds is included.
\end{abstract}

Key words: Chromates; langbeinites; lattice constants; sulfates; Tutton salts.

\section{Introduction}

The crystallographic data on langbeinite $\mathrm{A}_{2}^{+} \mathrm{B}_{2}^{2+}\left(\mathrm{XO}_{4}\right)_{3}$ and Tutton salt $\mathrm{A}_{2}^{+} \mathrm{B}^{2+}\left(\mathrm{XO}_{4}\right)_{2} \cdot 6 \mathrm{H}_{2} \mathrm{O}$ type compounds reported here were obtained as part of a project at the National Bureau of Standards ${ }^{1}$ in which $\mathrm{x}$-ray powder patterns of pure phases are prepared to extend and improve the Powder Diffraction File (PDF). ${ }^{2}$ Sulfates and chromates belonging to these groups of compounds, which are not represented in the PDF by good x-ray powder patterns, and which could be prepared with satisfactory purity, were studied. A literature search on other pertinent material was made and is summarized. Completely indexed x-ray patterns, optical properties, and a description of our methods are reported in the series NBS Monographs $25^{3}$ [41, 42, $43]^{4}$ for each phase studied. Briefly, the $x$-ray work was done on powders using a diffractometer, with an internal standard ( $\mathrm{Ag}$ or $\mathrm{W})$ for calibration of the $d$ spacing measurements.

The two groups of compounds are treated separately. In each group, the methods of preparation of the samples are outlined briefly, and tables summarizing our findings and those from the literature are given.

\subsection{Langbeinite Type Compounds}

\section{a. Structure and Preparation}

Langbeinite type compounds have the general formula $\mathrm{A}_{2}^{+} \mathrm{B}_{2}^{2+}\left(\mathrm{SO}_{4}\right)_{3}$. The prototype is $\mathrm{K}_{2} \mathrm{Mg}_{2}\left(\mathrm{SO}_{4}\right)_{3}$. The

\footnotetext{
*Research Associates of the Joint Committee on Powder Diffraction Standards.

${ }^{* *}$ Chemist. National Bureau of Standards.

${ }_{1}$ This project is sponsored in part by the Joint Committee on Powder Diffraction Standards.

${ }_{2}$ The Powder Diffraction File is compiled under the auspices of the Joint Committee on Powder Diffraction Standards. For information refer to the Joint Committee on Powder Powder Diffraction Standards. For information refer to the Joint
Diffraction Standards. 1601 Park Lane, Swarthmore, Pa. 19081.

Diffraction Standards. 1601 Park Lane, Swarthmore, Pa. 19081.
National Bureau of Standards Monograph 25, Standard X-ray Diffraction Patterns, Sec ${ }^{3}$ National Bureau of Standards Monograph 25 , Standard X-ray Diffraction Patterns, Sec.
$1-8$ and Circ. 539 , Sec. 1-10, have been published. They are available from the U.S. Government Printing Office, Washington, D.C. 20402.

${ }^{4}$ Figures in brackets indicate the literature references at the end of this paper.

${ }^{5} \mathrm{As}$ is customary in crystallographic studies, the cell parameters are given in terms of Angstrom $(\AA)$ equal to $10^{-10} \mathrm{~m}$
}

structure of this compound has been determined by Zemann and Zemann [64]. The basic structure is cubic with a tetramolecular unit cell and the space group $\mathrm{P} 2 / 3$ (No. 198). The $\mathrm{SO}_{4}$ tetrahedron is regular, and the $\mathrm{B}^{2+}$ ions are octahedrally coordinated. The $\mathrm{A}^{+}$ ions are of two kinds, one of which is surrounded by four oxygens at varying distances, and the other surrounded by three oxygens. Selenates or chromates of this structure have not prevjously been reported. The conditions for preparation of the langbeinite type compounds are given in table 1 . In this study four isostructural chromates were prepared, all with $\mathrm{B}^{2+}=\mathrm{Mg}^{2+}$. No attempt was made to prepare selenates. Two compounds, $\mathrm{K}_{2} \mathrm{Ca}_{2}\left(\mathrm{SO}_{4}\right)_{3}$ and $\mathrm{K}_{2} \mathrm{Cd}_{2}\left(\mathrm{SO}_{4}\right)_{3}$, were found to have orthorhombic distortions of the langbeinite structure.

\section{b. Results and Discussion}

Table 2 gives unit cell size, ${ }^{5}$ density, PDF card number if in the File and other relevant references. These data are given for the various langbeinite compounds from both our studies and from the literature. Two compounds were distorted from cubic and have been indexed as orthorhombic: data for these are given in table 3. It is of interest to note that Jona and Pepinsky [20] reported a transition in $\left(\mathrm{NH}_{4}\right)_{2} \mathrm{Cd} .2\left(\mathrm{SO}_{4}\right)_{3}$ at about $87 \mathrm{~K}$, doubtless to a distorted form. It is assumed that solid solutions will occur between most or all of these phases. The only studied solid solution here was between $\mathrm{K}_{2} \mathrm{M}_{2}\left(\mathrm{SO}_{4}\right)_{;}$; and $\mathrm{K}_{2} \mathrm{Ca}_{2}\left(\mathrm{SO}_{4}\right)_{\text {; }}$, the latter of which was orthorhombic. The compound midway between these end members was found to be cubic, confirming the findings of Morey et al. [36]. No langbeinites could be prepared with $\mathrm{Na}^{+}$as $\mathrm{A}$ and only a limited number with $\mathrm{Cs}^{+}$. Also none were prepared with cations larger than $\mathrm{Ca}^{2+}$ as $\mathrm{B}^{2+}$. It is of interest to note that the order of the cell size with constant $\mathrm{A}^{+}$is not in the one generally accepted as that of increasing radius of the $\mathrm{B}^{2+}$ 
TABLE 1. Preparation of langbeinite type compounds made at NBS

\begin{tabular}{|c|c|}
\hline Name & Method of preparation \\
\hline $\mathrm{K}_{2} \mathrm{Mg}_{2}\left(\mathrm{SO}_{4}\right)_{3}$ & Melting $\mathrm{K}_{2} \mathrm{SO}_{4}$ and $\mathrm{MgSO}_{4}$ together at about $1000^{\circ} \mathrm{C}$. The material is somewhat hygroscopic. \\
\hline $\mathrm{K}_{2} \mathrm{Ni}_{2}\left(\mathrm{SO}_{4}\right)_{3}$ & Heating a mixture of $\mathrm{NiSO}_{4}$ and $\mathrm{K}_{2} \mathrm{SO}_{4}$ at $750{ }^{\circ} \mathrm{C}$, then regrinding and annealing at $550{ }^{\circ} \mathrm{C}$ for $1 \frac{1 / 2}{\mathrm{hr}}$. \\
\hline $\mathrm{K}_{2} \mathrm{Co}_{2}\left(\mathrm{SO}_{4}\right)_{3}$ & Melting a mixture of $\mathrm{K}_{2} \mathrm{SO}_{4}$ and $\mathrm{CoSO}_{4}$ at about $600^{\circ} \mathrm{C}$. \\
\hline $\mathrm{K}_{2} \mathrm{Zn}_{2}\left(\mathrm{SO}_{4}\right)_{3}$ & Melting a mixture of $\mathrm{K}_{2} \mathrm{SO}_{4}$ and $\mathrm{ZnSO}_{4}$ and then grinding and annealing. \\
\hline $\mathrm{K}_{2} \mathrm{Mn}_{2}\left(\mathrm{SO}_{4}\right)_{3}$ & Melting a mixture of $\mathrm{K}_{2} \mathrm{SO}_{4}$ and $\mathrm{MnSO}_{4}$ cooling quickly and then annealing at $500{ }^{\circ} \mathrm{C}$ for $15 \mathrm{~h}$. \\
\hline $\mathrm{K}_{2} \mathrm{Ca}_{2}\left(\mathrm{SO}_{4}\right)_{3}$ & Melting a mixture of $\mathrm{K}_{2} \mathrm{SO}_{4}$ and $\mathrm{CaSO}_{4}$ and annealing for $18 \mathrm{~h}$ at $700{ }^{\circ} \mathrm{C}$. \\
\hline $\mathrm{K}_{2} \mathrm{Cd}_{2}\left(\mathrm{SO}_{4}\right)_{3}$ & Melting a mixture of $\mathrm{K}_{2} \mathrm{SO}_{4}$ and $\mathrm{CdSO}_{4}$ followed by annealing for $18 \mathrm{~h}$ at $300{ }^{\circ} \mathrm{C}$ then 3 days at $150{ }^{\circ} \mathrm{C}$. \\
\hline $\mathrm{K}_{2} \mathrm{MgCa}\left(\mathrm{SO}_{4}\right)_{3}$ & Melting a mixture of $\mathrm{K}_{2} \mathrm{SO}_{4}, \mathrm{MgSO}_{4}$ and $\mathrm{CaSO}_{4}$ followed by annealing for $20 \mathrm{~h}$ at $500{ }^{\circ} \mathrm{C}$ then $17 \mathrm{~h}$ at $400{ }^{\circ} \mathrm{C}$. \\
\hline$\left(\mathrm{NH}_{4}\right)_{2} \mathrm{Mn}_{2}\left(\mathrm{SO}_{4}\right)_{3}$ & Partial evaporation at $100{ }^{\circ} \mathrm{C}$ of a $1: 2$ aqueous solution of $\left(\mathrm{NH}_{4}\right)_{2} \mathrm{SO}_{4}$ and $\mathrm{MnSO}_{4}$. \\
\hline$\left(\mathrm{NH}_{4}\right)_{2} \mathrm{Cd}_{2}\left(\mathrm{SO}_{4}\right)_{3}$ & $\begin{array}{l}\text { Partial evaporation at } 90^{\circ} \mathrm{C} \text { of a } 1: 2 \text { aqueous solution of }\left(\mathrm{NH}_{4}\right)_{2} \mathrm{SO}_{4} \text { and } \mathrm{CdSO}_{4} \text {. The crystals were then quickly } \\
\text { washed with hot water and alcohol. }\end{array}$ \\
\hline$\left(\mathrm{NH}_{4}\right)_{2} \mathrm{Ca}\left(\mathrm{SO}_{4}\right)_{3}$ & $\begin{array}{l}\text { Heating near } 100{ }^{\circ} \mathrm{C} \text { a mixture of } 2.7 \text { percent } \mathrm{CaSO}_{4}, 41.1 \text { percent }\left(\mathrm{NH}_{4}\right)_{2} \mathrm{SO}_{4} \text { and } 56.2 \text { percent } \mathrm{H}_{2} \mathrm{O} \text { (by wt) for } 5 \mathrm{~h} \\
\text { using phase study information of Hill and Yanick [15]. The slurry was filtered off rapidly. }\end{array}$ \\
\hline $\mathrm{Tl}_{2} \mathrm{Co}_{2}\left(\mathrm{SO}_{4}\right)_{3}$ & Heating $\mathrm{Tl}_{2} \mathrm{SO}_{4}$ and $\mathrm{CoSO}_{4}$ together for several hours at $500^{\circ} \mathrm{C}$. \\
\hline $\mathrm{Tl}_{2} \mathrm{Mn}_{2}\left(\mathrm{SO}_{4}\right)_{3}$ & Slow evaporation at room temperature of a $1: 2$ aqueous solution of $\mathrm{Tl}_{2} \mathrm{SO}_{4}$ and $\mathrm{MnSO}_{4}$. \\
\hline $\mathrm{Tl}_{2} \mathrm{Cd}_{2}\left(\mathrm{SO}_{4}\right)_{3}$ & Heating a mixture of $\mathrm{Tl}_{2} \mathrm{SO}_{4}$ and $\mathrm{CdSO}_{4}$ for $100 \mathrm{~h}$ at $470^{\circ}$; followed by regrinding and reheating. \\
\hline $\mathrm{Rb}_{2} \mathrm{Mg}_{2}\left(\mathrm{SO}_{4}\right)_{3}$ & Melting a mixture of $\mathrm{Rb}_{2} \mathrm{SO}_{4}$ and $\mathrm{MgSO}_{4}$, cooling quickly then annealing at $800{ }^{\circ} \mathrm{C}$ for $18 \mathrm{~h}$. \\
\hline $\mathrm{Rb}_{2} \mathrm{Ni}_{2}\left(\mathrm{SO}_{4}\right)_{3}$ & Heating $\mathrm{Rb}_{2} \mathrm{SO}_{4}$ and $\mathrm{NiSO}_{4}$ together in $\mathrm{N}_{2}$ for $10 \mathrm{~min}$, regrinding, and reheating at $450^{\circ}$ for $15 \mathrm{~min}$. \\
\hline $\mathrm{Rb}_{2} \mathrm{Co}_{2}\left(\mathrm{SO}_{4}\right)_{2}$ & Heating $\mathrm{Rb}_{2} \mathrm{SO}_{4}$ and $\mathrm{CoSO}_{4}$ together in $\mathrm{N}_{2}$ at $540^{\circ} \mathrm{C}$ for $10 \mathrm{~min}$, then grinding and reheating for $10 \mathrm{~min}$ at $540{ }^{\circ} \mathrm{C}$. \\
\hline $\mathrm{Rb}_{2} \mathrm{Mn}_{2}\left(\mathrm{SO}_{4}\right)_{3}$ & Melting a mixture of $\mathrm{Rb}_{2} \mathrm{SO}_{4}$ and $\mathrm{MnSO}_{4}$. \\
\hline $\mathrm{Rb}_{2} \mathrm{Cd}_{2}\left(\mathrm{SO}_{4}\right)_{3}$ & Melting a mixture of $\mathrm{Rb}_{2} \mathrm{SO}_{4}$ and $\mathrm{CdSO}_{4}$, quenching, grinding, and annealing for $18 \mathrm{~h}$ at $700{ }^{\circ} \mathrm{C}$. \\
\hline $\mathrm{Rb}_{2} \mathrm{Ca}_{2}\left(\mathrm{SO}_{4}\right)_{3}$ & Melting a mixture of $\mathrm{Rb}_{2} \mathrm{SO}_{4}$ and $\mathrm{CaSO}_{4}$, quenching, grinding and annealing at $650{ }^{\circ} \mathrm{C}$ for several days. \\
\hline $\mathrm{Cs}_{2} \mathrm{Ca}_{2}\left(\mathrm{SO}_{4}\right)_{3}$ & Melting a mixture of $\mathrm{Cs}_{2} \mathrm{SO}_{4}$ and $\mathrm{CaSO}_{4}$, quenching and annealing for $18 \mathrm{~h}$ at $700{ }^{\circ} \mathrm{C}$. \\
\hline $\mathrm{K}_{2} \mathrm{Mg}_{2}\left(\mathrm{CrO}_{4}\right)_{3}$ & Heating $\mathrm{K}_{2} \mathrm{CrO}_{4}$ and $\mathrm{MgCrO}_{4}$ together for one half hour at $510^{\circ} \mathrm{C}$ in $\mathrm{N}_{2}$. \\
\hline $\mathrm{Rb}_{2} \mathrm{Mg}_{2}\left(\mathrm{CrO}_{4}\right)_{3}$ & Heating $\mathrm{Rb}_{2} \mathrm{CrO}_{4}$ and $\mathrm{MgCrO}_{4}$ together for $45 \mathrm{~min}$ at $500{ }^{\circ} \mathrm{C}$ in $\mathrm{N}_{2}$. \\
\hline $\mathrm{Tl}_{2} \mathrm{Mg}_{2}\left(\mathrm{CrO}_{4}\right)_{3}$ & Heating $\mathrm{Tl}_{2} \mathrm{CrO}_{4}$ and $\mathrm{MgCrO}_{4}$ together for $\mathrm{l} \mathrm{h}$ at $380^{\circ}$. \\
\hline $\mathrm{Cs}_{2} \mathrm{Mg}_{2}\left(\mathrm{CrO}_{4}\right)_{3}$ & Heating $\mathrm{Cr}_{2} \mathrm{CrO}_{4}$ and $\mathrm{MgCrO}_{4}$ together for $25 \mathrm{~min}$ at $440^{\circ}$ in $\mathrm{N}_{2}$. \\
\hline
\end{tabular}

TABLE 2. Langbeinite type sulfates-cubic, $P 2{ }_{1} 3$ (No. 198)

\begin{tabular}{|c|c|c|c|c|c|}
\hline \multirow{2}{*}{ Compound } & \multicolumn{2}{|c|}{ Cell size } & \multirow{2}{*}{$\begin{array}{c}\text { Density } \\
\mathrm{g} / \mathrm{cm}^{3}\end{array}$} & \multirow{2}{*}{$\mathrm{PDF}^{\mathrm{b}} \mathrm{N}_{0}$. } & \multirow{2}{*}{ Other Ref. } \\
\hline & $\AA$ & Ref. & & & \\
\hline 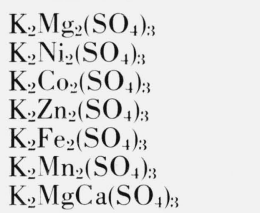 & $\begin{array}{c}9.9211 \\
9.8436 \\
9.9313 \\
9.9247 \\
10.007 \\
10.1143 \\
10.1662\end{array}$ & $\begin{array}{l}41 \\
41 \\
41 \\
41 \\
10 \\
41 \\
42\end{array}$ & $\begin{array}{l}2.823 \\
3.369 \\
3.283 \\
3.376 \\
3.168 \\
3.057 \\
2.723\end{array}$ & $\begin{array}{l}19-974 \\
19-984 \\
20-873 \\
20-957 \\
18-1027 \\
20-909 \\
20-866\end{array}$ & $\begin{array}{l}7,10,36,64 \\
10 \\
10 \\
10 \\
3,10 \\
36\end{array}$ \\
\hline $\begin{array}{l}\mathrm{K}_{2} \mathrm{Cd}_{2}\left(\mathrm{SO}_{4}\right)_{3} \\
\mathrm{~K}_{2} \mathrm{Ca}_{2}\left(\mathrm{SO}_{4}\right)_{3}\end{array}$ & $\begin{array}{l}(\mathrm{a}) \\
\left({ }^{\mathrm{a}}\right)\end{array}$ & & & & \\
\hline $\begin{array}{l}\left(\mathrm{NH}_{4}\right)_{2} \mathrm{Mg}_{2}\left(\mathrm{SO}_{4}\right)_{3} \\
\left(\mathrm{NH}_{4}\right)_{2} \mathrm{Ni}_{2}\left(\mathrm{SO}_{4}\right)_{3} \\
\left(\mathrm{NH}_{4}\right)_{2} \mathrm{Co}_{2}\left(\mathrm{SO}_{4}\right)_{3} \\
\left(\mathrm{NH}_{4}\right)_{2} \mathrm{Fe}_{2}\left(\mathrm{SO}_{4}\right)_{3} \\
\left(\mathrm{NH}_{4}\right)_{2} \mathrm{Mn}_{2}\left(\mathrm{SO}_{4}\right)_{3} \\
\left(\mathrm{NH}_{4}\right)_{2} \mathrm{Cd} \cdot\left(\mathrm{SO}_{4}\right)_{3} \\
\left(\mathrm{NH}_{4}\right)_{2} \mathrm{Ca}_{2}\left(\mathrm{SO}_{4}\right)_{3}\end{array}$ & $\begin{array}{l}9.99 \\
9.914 \\
9.95 \\
10.09 \\
10.1892 \\
10.3511 \\
10.5360\end{array}$ & $\begin{array}{l}22 \\
22 \\
22 \\
22 \\
42 \\
42 \\
43\end{array}$ & $\begin{array}{l}2.248 \\
3.011 \\
2.981 \\
2.819 \\
2.726 \\
3.288 \\
2.297\end{array}$ & $\begin{array}{l}18-110 \\
18-120 \\
18-85 \\
18-105 \\
20-96 \\
21-791 \\
11-241\end{array}$ & $\begin{array}{l}10 \\
10 \\
10 \\
10 \\
10,20 \\
10,15\end{array}$ \\
\hline $\begin{array}{l}\mathrm{Tl}_{2} \mathrm{Co}_{2}\left(\mathrm{SO}_{4}\right)_{3} \\
\mathrm{Tl}_{2} \mathrm{Fe}_{2}\left(\mathrm{SO}_{4}\right)_{3} \\
\mathrm{Tl}_{2} \mathrm{Mn}_{2}\left(\mathrm{SO}_{4}\right)_{3} \\
\mathrm{Tl}_{2} \mathrm{Cd}_{2}\left(\mathrm{SO}_{4}\right)_{3}\end{array}$ & $\begin{array}{l}10.0312 \\
10.108 \\
10.2236 \\
10.3841\end{array}$ & $\begin{array}{l}43 \\
10 \\
42 \\
43\end{array}$ & $\begin{array}{l}5.361 \\
5.200 \\
5.015 \\
5.467\end{array}$ & $\begin{array}{l}22-1466 \\
20-1263 \\
22-1465\end{array}$ & $\begin{array}{l}10 \\
10\end{array}$ \\
\hline $\begin{array}{l}\mathrm{Rb}_{2} \mathrm{M}_{\mathrm{g}_{2}}\left(\mathrm{SO}_{4}\right)_{3} \\
\mathrm{Rb}_{2} \mathrm{Ni}_{2}\left(\mathrm{SO}_{4}\right)_{3} \\
\mathrm{Rb}_{2} \mathrm{Co}_{2}\left(\mathrm{SO}_{4}\right)_{3} \\
\mathrm{Rb}_{2} \mathrm{Fe}_{2}\left(\mathrm{SO}_{4}\right)_{3} \\
\mathrm{Rb}_{2}, \mathrm{Mn}_{2}\left(\mathrm{SO}_{4}\right)_{3} \\
\mathrm{Rb}_{2} \mathrm{Cd}_{2}\left(\mathrm{SO}_{4}\right)_{3} \\
\mathrm{Rb}_{2} \mathrm{Ca}_{2}\left(\mathrm{SO}_{4}\right)_{3}\end{array}$ & $\begin{array}{l}10.0051 \\
9.9217 \\
10.0204 \\
10.098 \\
10.2147 \\
10.3810 \\
10.5687\end{array}$ & $\begin{array}{l}42 \\
43 \\
43 \\
10 \\
42 \\
42 \\
42\end{array}$ & $\begin{array}{l}3.367 \\
3.921 \\
3.807 \\
3.682 \\
3.546 \\
4.060 \\
3.034\end{array}$ & $\begin{array}{l}21-1029 \\
22-1284 \\
22-1266 \\
20-1000 \\
20-1022 \\
20-1023\end{array}$ & $\begin{array}{l}10 \\
10 \\
10 \\
10 \\
10 \\
10\end{array}$ \\
\hline $\mathrm{Cs}_{2} \mathrm{Ca}_{2}\left(\mathrm{SO}_{4}\right)_{3}$ & 10.7213 & 42 & 3.417 & $20-275$ & 10 \\
\hline
\end{tabular}

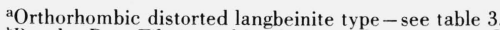

bowder Data File issued by the Joint Committee on Powder Diffraction Standards. 
TABLE 3. Langbeinite type sulfates-orthorhombic, $\mathrm{P} 2_{1} 2_{1} 2_{1}$ (No. 19)

\begin{tabular}{|c|c|c|c|c|c|c|c|}
\hline \multirow{2}{*}{ Compound } & \multicolumn{3}{|c|}{ Cell size } & \multirow{2}{*}{$\begin{array}{l}\text { Refer- } \\
\text { ence }\end{array}$} & \multirow{2}{*}{$\begin{array}{c}\text { Density } \\
y / \mathrm{cm}^{3}\end{array}$} & \multirow{2}{*}{$\begin{array}{l}\mathrm{PDF}^{\mathrm{a}} \\
\mathrm{N}_{0} .\end{array}$} & \multirow{2}{*}{$\begin{array}{l}\text { Other } \\
\text { reference }\end{array}$} \\
\hline & $a(\AA)$ & $b(\AA)$ & $c(\AA)$ & & & & \\
\hline $\begin{array}{l}\mathrm{K}_{2} \mathrm{Cd}_{2}\left(\mathrm{SO}_{4}\right):: \\
\mathrm{K}_{2} \mathrm{Ca}_{2}\left(\mathrm{SO}_{1}\right)::\end{array}$ & $\begin{array}{l}10.212 \\
10.334\end{array}$ & $\begin{array}{l}10.280 \\
10.501\end{array}$ & $\begin{array}{l}10.171 \\
10.186\end{array}$ & $\begin{array}{l}42 \\
42\end{array}$ & $\begin{array}{l}3.677 \\
2.683\end{array}$ & $\begin{array}{l}20-864 \\
20-867\end{array}$ & $\begin{array}{c}10 \\
\text { 4. } 36.40\end{array}$ \\
\hline
\end{tabular}

ions $(\mathrm{Mg}<\mathrm{Ni}<\mathrm{Co}<\mathrm{Zn}<\mathrm{Fe})$. The chromate langbeinites are reported in table 4.

\subsection{Tutton Salts}

\section{a. Structure}

The Tutton salts have the general composition $\mathrm{A}^{+} \mathrm{B}^{2+}\left(\mathrm{XO}_{4}\right)_{2} \cdot 6 \mathrm{H}_{2} \mathrm{O}$. These salts were extensively studied by the goniometric method around the turn of the century by Tutton [45-62] and proved to be an early example of a large isostructural series. The structure of one member of the series was determined by Margulis and Templeton [29] and was found to be in the space group $\mathrm{P} 2{ }_{1} / \mathrm{a}$ (No. 14) with two molecules per cell. The $\mathrm{B}^{2+}$ ions are octahedrally surrounded by water molecules, and the $\mathrm{A}^{+}$is bonded to oxygens of the sulfate tetrahedron and water oxygens. The structure reported earlier by Hofmann [16] was found incorrect [29].

Tutton found a large number of similar selenates [57-61] and three chromates were also reported in this series [62]; in all of these $\mathrm{B}^{2+}$ was $\mathrm{Mg}$. In the present study one new chromate was made in which $\mathrm{B}^{2+}$ was $\mathrm{Ni}$. A series of isostructural fluoberyllates has also been reported $[37,38]$.

\section{b. Preparation}

Most of the materials made here were prepared by slow evaporation at room temperature of $1: 1$ aqueous solution of $\mathrm{A}_{2} \mathrm{SO}_{4}$ and $\mathrm{BSO}_{4}$. The phases which were prepared differently are discussed below:

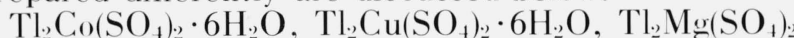
- $6 \mathrm{H}_{2} \mathrm{O}$ and $\mathrm{Tl}_{2} \mathrm{Zn}\left(\mathrm{SO}_{4}\right)_{2} \cdot 6 \mathrm{H}_{2} \mathrm{O}$ were made by slow evaporation at room temperature of an 1:8 aqueous

TABLE 4. Langbeinite type chromates, cubic P2,3 (No. 198)

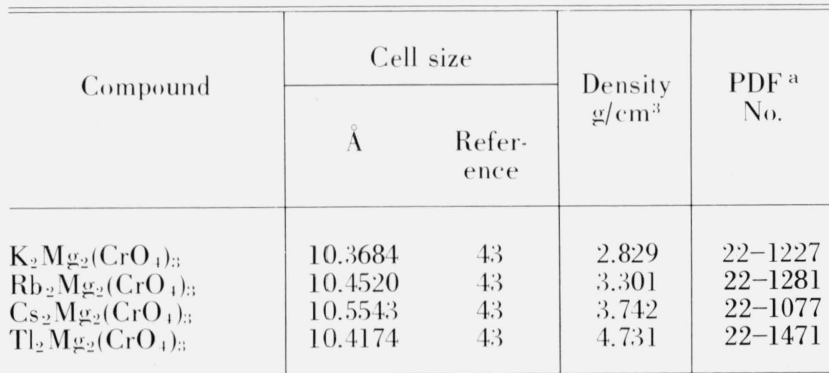

Powder Data File issued by the Joint Committee on Powder Diffraction Standards. solution of $\mathrm{Tl}_{2} \mathrm{SO}_{4}$ and the relevant $\mathrm{BSO}_{4}$. The first crystals formed were used.

$\mathrm{Cs} \cdot 2 \mathrm{Mg}(\mathrm{CrO})_{2} \cdot 6 \mathrm{H}_{2} \mathrm{O}, \quad \mathrm{K}_{2} \mathrm{Mg}_{2}\left(\mathrm{SO}_{4}\right)_{2} \cdot 6 \mathrm{H}_{2} \mathrm{O}$, and $\mathrm{Rb}{ }_{2} \mathrm{Fe}\left(\mathrm{SO}_{4}\right)_{2} \cdot 6 \mathrm{H}_{2} \mathrm{O}$ were made by slow evaporation at room temperature of a $1: 3$ aqueous solution of the relevant $\mathrm{A}_{2} \mathrm{XO}_{4}$ and $\mathrm{BXO}_{4}$. The first crystals formed were used.

$\mathrm{Tl}_{2} \mathrm{Mn}\left(\mathrm{SO}_{4}\right)_{2} \cdot 6 \mathrm{H}_{2} \mathrm{O}$ was made by evaporatine at about $90{ }^{\circ} \mathrm{C}$ a $1: 1$ aqueous solution of $\mathrm{Tl}_{2} \mathrm{SO}_{4}$ and $\mathrm{MnSO}_{4}$. The crystals were filtered off while hot.

$\mathrm{Tl}_{2} \mathrm{Fe}\left(\mathrm{SO}_{4}\right)_{2} \cdot 6 \mathrm{H}_{2} \mathrm{O}$ was made by slow evaporation at room temperature of a $1: 1$ solution of $\mathrm{Tl}_{2} \mathrm{SO}_{4}$ and $\mathrm{FeSO}_{4}$. The first crystals formed were used.

$\mathrm{Rb}_{2} \mathrm{Cd}\left(\mathrm{SO}_{4}\right)_{2} \cdot 6 \mathrm{H}_{2} \mathrm{O}$ and $\mathrm{Cs}_{2} \mathrm{Cd}\left(\mathrm{SO}_{4}\right)_{2} \cdot 6 \mathrm{H}_{2} \mathrm{O}$ were too unstable to study, due to loss of water. The $\left(\mathrm{NH}_{4}\right)_{2} \mathrm{Cs}\left(\mathrm{SO}_{4}\right)_{2} \cdot 6 \mathrm{H}_{2} \mathrm{O}$ and $\left(\mathrm{NH}_{4}\right)_{2} \mathrm{Mn}\left(\mathrm{SO}_{4}\right)_{2} \cdot 6 \mathrm{H}_{2} \mathrm{O}$ tended to lose water in dry air and required special handling.

\section{c. Results}

In tables 5 and 6 are given the cell parameters from our studies [41, 42, 43] or from the indicated literature together with the cell volume and density. The PDF numbers are given when available, and references to other studies on the materials are indicated.

No Tutton salts are known with $\mathrm{Na}^{+}$as $\mathrm{A}^{+}$, or with cations larger than $\mathrm{Ca}^{2+}$ as $\mathrm{B}^{2+}$. As with the langbeinite series the volume of the cell with constant $\mathrm{A}^{+}$and with different $\mathrm{B}^{2+}$ ions is not in the accepted order of the ionic radii of the $\mathrm{B}^{2+}$ ions $(\mathrm{M} g<\mathrm{Ni}<\mathrm{Co}<\mathrm{Zn}<\mathrm{Fe})$.

\section{References}

[1] Bakumskaya, E. L., and Bergman, A. G., Russ. J. Inorg. Chem. (Eng. Trans.) 1, 1035 (1956).

[2] Barker, T. V., J. Chem. Soc. 99, 1326 (1911).

[3] Bellanca, A., Accad. dei Lincei Renconti, Classe Scieza, Serces 8, 2,451 (1947).

[4] Bellanca, A., Periodico Mineral, (Rome) 13,21 (1942).

[5] Bhowmik, G., Indian J. Phys. 39, 307 (1965).

[6] Carapezza, M., and diSansererino, C., Mineral. Petrograph Acta 13, 321 (1967).

[7] Cipriani, C., Rend. Soc., Mineral, Italiano 14, 3 (1958).

[8] DeWolff, P. M., Techn. Phy. Dienst., Delft, Holland, in Powder Data File.

[9] Garvanelli, C. L., Lincei Rend. Sc. Fis. Mat. i. Nat. 36, 524 (1964).

[10] Gattow, G., and Zemann, J., Z. Anorg. Allgem. Chem. 293, 233 (1958).

[11] Gossner, B., and Koch. J., Z. Krist. 80, 455 (1931).

[12 Grimes, N. W., Kay, H. F., and Webb, M. W., Acta Cryst. 16 , 823 (1963).

[13] Halla, F., and Mehl, E., Z. Anorg. Allgem. Chem. 199, 379 (1931).

[14] Hartman, P., and Woensdregt, C. F., Acta Cryst 1 7, 779 (1964). 
TABLE 5. Tutton salts-sulfates, monoclinic, $\mathrm{P} 2_{1} / \mathrm{a}$ (No. 14)

\begin{tabular}{|c|c|c|c|c|c|c|c|c|c|}
\hline \multirow{2}{*}{ Compound } & \multicolumn{4}{|c|}{ Cell size } & \multirow{2}{*}{$\begin{array}{l}\text { Vol. } \\
\left(\AA^{3}\right)\end{array}$} & \multirow{2}{*}{$\begin{array}{l}\text { Refer- } \\
\text { ence }\end{array}$} & \multirow{2}{*}{$\begin{array}{l}\text { Density } \\
\mathrm{g} / \mathrm{cm}^{3}\end{array}$} & \multirow{2}{*}{$\begin{array}{l}\mathrm{PDF}^{\mathrm{a}} \\
\text { No. }\end{array}$} & \multirow{2}{*}{$\begin{array}{l}\text { Other } \\
\text { references }\end{array}$} \\
\hline & $a(\AA)$ & $b(\AA)$ & $c(\AA)$ & $\beta$ & & & & & \\
\hline $\begin{array}{l}\mathrm{K}_{2} \mathrm{Mg}\left(\mathrm{SO}_{4}\right)_{2} \cdot 6 \mathrm{H}_{2} \mathrm{O} \\
\mathrm{K}_{2} \mathrm{Ni}\left(\mathrm{SO}_{4}\right)_{2} \cdot 6 \mathrm{H}_{2} \mathrm{O}\end{array}$ & $\begin{array}{l}9.096 \\
8.991\end{array}$ & $\begin{array}{l}12.254 \\
12.184\end{array}$ & $\begin{array}{l}6.128 \\
6.130\end{array}$ & $\begin{array}{l}104^{\circ} 47^{\prime} \\
105^{\circ}\end{array}$ & $\begin{array}{l}660.50 \\
648.40\end{array}$ & $\begin{array}{l}22 \\
63\end{array}$ & $\begin{array}{l}2.051 \\
2.24\end{array}$ & $\begin{array}{l}20-839 \\
13-193\end{array}$ & $\begin{array}{l}23,48 \\
48\end{array}$ \\
\hline $\begin{array}{l}\mathrm{K}_{2} \mathrm{Cu}\left(\mathrm{SO}_{4}\right)_{2} \cdot 6 \mathrm{H}_{2} \mathrm{O} \\
\mathrm{K}_{2} \mathrm{Co}\left(\mathrm{SO}_{4}\right)_{2} \cdot 6 \mathrm{H}_{2} \mathrm{O}\end{array}$ & $\begin{array}{l}9.09 \\
9.059\end{array}$ & $\begin{array}{l}12.14 \\
12.209\end{array}$ & $\begin{array}{l}6.18 \\
6.152\end{array}$ & $\begin{array}{l}104^{\circ} 28^{\prime} \\
104^{\circ} 48^{\prime}\end{array}$ & $\begin{array}{l}660.35 \\
657.77\end{array}$ & $\begin{array}{r}22 \\
8\end{array}$ & $\begin{array}{l}2.223 \\
2.207\end{array}$ & $\begin{array}{l}17-137 \\
21-632\end{array}$ & $\begin{array}{l}23,48 \\
14,23\end{array}$ \\
\hline $\begin{array}{l}\mathrm{K}_{2} \mathrm{Zn}\left(\mathrm{SO}_{4}\right)_{2} \cdot 6 \mathrm{H}_{2} \mathrm{O} \\
\mathrm{K}_{2} \mathrm{Fe}\left(\mathrm{SO}_{4}\right)_{2} \cdot 6 \mathrm{H}_{2} \mathrm{O}\end{array}$ & $\begin{array}{l}9.041 \\
9.05\end{array}$ & $\begin{array}{l}12.215 \\
12.27\end{array}$ & $\begin{array}{l}6.156 \\
6.16\end{array}$ & $\begin{array}{l}104^{\circ} 49^{\prime} \\
104^{\circ} 32^{\prime}\end{array}$ & $\begin{array}{l}657.18 \\
662.14\end{array}$ & $\begin{array}{l}42 \\
22\end{array}$ & $\begin{array}{l}2.242 \\
2.178\end{array}$ & $\begin{array}{l}20-958 \\
17-486\end{array}$ & $\begin{array}{l}23,48 \\
23,48\end{array}$ \\
\hline $\begin{array}{l}\mathrm{Tl}_{2} \mathrm{Mg}\left(\mathrm{SO}_{4}\right)_{2} \cdot 6 \mathrm{H}_{2} \mathrm{O} \\
\mathrm{Tl}_{2} \mathrm{Ni}\left(\mathrm{SO}_{4}\right)_{2} \cdot 6 \mathrm{H}_{2} \mathrm{O}\end{array}$ & $\begin{array}{l}9.273 \\
9.166\end{array}$ & $\begin{array}{l}12.472 \\
12.392\end{array}$ & $\begin{array}{l}6.214 \\
6.216\end{array}$ & $\begin{array}{l}106^{\circ} 23^{\prime} \\
106^{\circ} 20^{\prime}\end{array}$ & $\begin{array}{l}689.49 \\
677.55\end{array}$ & $\begin{array}{l}42 \\
42\end{array}$ & $\begin{array}{l}3.532 \\
3.763\end{array}$ & $\begin{array}{l}21-1243 \\
20-1266\end{array}$ & $\begin{array}{l}18,53 \\
6,56\end{array}$ \\
\hline $\begin{array}{l}\mathrm{Tl}_{2} \mathrm{Cu}\left(\mathrm{SO}_{4}\right)_{2} \cdot 6 \mathrm{H}_{2} \mathrm{O} \\
\mathrm{Tl}_{2} \mathrm{Co}\left(\mathrm{SO}_{4}\right)_{2} \cdot 6 \mathrm{H}_{2} \mathrm{O}\end{array}$ & $\begin{array}{l}9.268 \\
9.235\end{array}$ & $\begin{array}{l}12.364 \\
12.442\end{array}$ & $\begin{array}{l}6.216 \\
6.227\end{array}$ & $\begin{array}{l}105^{\circ} 33^{\prime} . \\
106^{\circ} 24^{\prime}\end{array}$ & $\begin{array}{l}686.22 \\
686.38\end{array}$ & $\begin{array}{l}42 \\
42\end{array}$ & $\begin{array}{l}3.740 \\
3.7152\end{array}$ & $\begin{array}{l}21-1412 \\
21-1411\end{array}$ & $\begin{array}{l}53 \\
53\end{array}$ \\
\hline $\begin{array}{l}\mathrm{Tl}_{2} \mathrm{Zn}\left(\mathrm{SO}_{4}\right)_{2} \cdot 6 \mathrm{H}_{2} \mathrm{O} \\
\mathrm{Tl}_{2} \mathrm{Fe}\left(\mathrm{SO}_{4}\right)_{2} \cdot 6 \mathrm{H}_{2} \mathrm{O}\end{array}$ & $\begin{array}{l}9.219 \\
9.264\end{array}$ & $\begin{array}{l}12.433 \\
12.499\end{array}$ & $\begin{array}{l}6.2317 \\
6.236\end{array}$ & $\begin{array}{l}106^{\circ} 18^{\prime} \\
106^{\circ} 9^{\prime}\end{array}$ & $\begin{array}{l}685.58 \\
693.51\end{array}$ & $\begin{array}{l}42 \\
43\end{array}$ & $\begin{array}{l}3.763 \\
3.662\end{array}$ & $\begin{array}{l}22-1244 \\
22-921\end{array}$ & $\begin{array}{l}54 \\
53\end{array}$ \\
\hline $\begin{array}{l}\left(\mathrm{NH}_{4}\right)_{2} \mathrm{Mg}\left(\mathrm{SO}_{4}\right)_{2} \cdot 6 \mathrm{H}_{2} \mathrm{O} \\
\left(\mathrm{NH}_{4}\right)_{2} \mathrm{Ni}\left(\mathrm{SO}_{4}\right)_{2} \cdot 6 \mathrm{H}_{2} \mathrm{O}\end{array}$ & $\begin{array}{l}9.324 \\
9.19\end{array}$ & $\begin{array}{l}12.597 \\
12.47\end{array}$ & $\begin{array}{l}6.211 \\
6.24\end{array}$ & $\begin{array}{l}107^{\circ} 14^{\prime} \\
106^{\circ} 46^{\prime}\end{array}$ & $\begin{array}{l}696.76 \\
684.70\end{array}$ & $\begin{array}{l}22 \\
12\end{array}$ & $\begin{array}{l}1.718 \\
1.913\end{array}$ & $\begin{array}{l}17-135 \\
12-454\end{array}$ & $\begin{array}{l}7,29,30,24,47 \\
12,24,32,50\end{array}$ \\
\hline $\begin{array}{l}\left(\mathrm{NH}_{4}\right)_{2} \mathrm{Cu}\left(\mathrm{SO}_{4}\right)_{2} \cdot 6 \mathrm{H}_{2} \mathrm{O} \\
\left(\mathrm{NH}_{4}\right)_{2} \mathrm{Co}\left(\mathrm{SO}_{4}\right)_{2} \cdot 6 \mathrm{H}_{2} \mathrm{O}\end{array}$ & $\begin{array}{l}9.206 \\
9.258\end{array}$ & $\begin{array}{l}12.38 \\
12.528\end{array}$ & $\begin{array}{l}6.299 \\
6.236\end{array}$ & $\begin{array}{l}106^{\circ} 12^{\prime} \\
107^{\circ} 6^{\prime}\end{array}$ & $\begin{array}{l}689.39 \\
697.98\end{array}$ & $\begin{array}{l}8 \\
8\end{array}$ & $\begin{array}{l}1.924 \\
1.897\end{array}$ & $\begin{array}{l}11-660 \\
20-771\end{array}$ & $\begin{array}{l}5,14,24,34,50 \\
13,14,24,50\end{array}$ \\
\hline $\begin{array}{l}\left(\mathrm{NH}_{4}\right)_{2} \mathrm{Zn}\left(\mathrm{SO}_{4}\right)_{2} \cdot 6 \mathrm{H}_{2} \mathrm{O} \\
\left(\mathrm{NH}_{4}\right)_{2} \mathrm{Fe}\left(\mathrm{SO}_{4}\right)_{2} \cdot 6 \mathrm{H}_{2} \mathrm{O}\end{array}$ & $\begin{array}{l}9.205 \\
9.28\end{array}$ & $\begin{array}{l}12.475 \\
12.57\end{array}$ & $\begin{array}{l}6.225 \\
6.22\end{array}$ & $\begin{array}{l}106^{\circ} 52^{\prime} \\
106^{\circ} 50^{\prime}\end{array}$ & $\begin{array}{l}684.08 \\
694.47\end{array}$ & $\begin{array}{l}22 \\
22\end{array}$ & $\begin{array}{l}1.950 \\
1.875\end{array}$ & $\begin{array}{l}18-138 \\
17-481\end{array}$ & $\begin{array}{l}24,33,47 \\
9,24,31,55\end{array}$ \\
\hline $\begin{array}{l}\left(\mathrm{NH}_{4}\right)_{2} \mathrm{Mn}\left(\mathrm{SO}_{4}\right)_{2} \cdot 6 \mathrm{H}_{2} \mathrm{O} \\
\left(\mathrm{NH}_{4}\right)_{2} \mathrm{Cd}\left(\mathrm{SO}_{4}\right)_{2} \cdot 6 \mathrm{H}_{2} \mathrm{O}\end{array}$ & $\begin{array}{l}9.374 \\
9.393\end{array}$ & $\begin{array}{l}12.676 \\
12.777\end{array}$ & $\begin{array}{l}6.253 \\
6.300\end{array}$ & $\begin{array}{l}106^{\circ} 49^{\prime} \\
106^{\circ} 42^{\prime}\end{array}$ & $\begin{array}{l}711.21 \\
724.17\end{array}$ & $\begin{array}{l}43 \\
43\end{array}$ & $\begin{array}{l}1.827 \\
2.058\end{array}$ & $11-134$ & $\begin{array}{l}7,24,30 \\
35,50\end{array}$ \\
\hline $\begin{array}{l}\mathrm{Rb}_{2} \mathrm{Mg}\left(\mathrm{SO}_{4}\right)_{2} \cdot 6 \mathrm{H}_{2} \mathrm{O} \\
\mathrm{Rb}_{2} \mathrm{Ni}\left(\mathrm{SO}_{4}\right)_{2} \cdot 6 \mathrm{H}_{2} \mathrm{O}\end{array}$ & $\begin{array}{l}9.235 \\
9.138\end{array}$ & $\begin{array}{l}12.486 \\
12.416\end{array}$ & $\begin{array}{l}6.224 \\
6.223\end{array}$ & $\begin{array}{l}105^{\circ} 59^{\prime} \\
106^{\circ} 4^{\prime}\end{array}$ & $\begin{array}{l}689.85 \\
678.47\end{array}$ & $\begin{array}{l}43 \\
43\end{array}$ & $\begin{array}{l}2.385 \\
2.594\end{array}$ & $\begin{array}{l}21-1415 \\
21-1418\end{array}$ & $\begin{array}{l}48 \\
48\end{array}$ \\
\hline $\begin{array}{l}\mathrm{Rb}_{2} \mathrm{Cu}\left(\mathrm{SO}_{4}\right)_{2} \cdot 6 \mathrm{H}_{2} \mathrm{O} \\
\mathrm{Rb}_{2} \mathrm{Co}\left(\mathrm{SO}_{4}\right)_{2} \cdot 6 \mathrm{H}_{2} \mathrm{O}\end{array}$ & $\begin{array}{l}9.267 \\
9.180\end{array}$ & $\begin{array}{l}12.366 \\
12.433\end{array}$ & $\begin{array}{l}6.228 \\
6.230\end{array}$ & $\begin{array}{l}105^{\circ} 19^{\prime} \\
106^{\circ} 1^{\prime}\end{array}$ & $\begin{array}{l}688.35 \\
683.46\end{array}$ & $\begin{array}{l}43 \\
14\end{array}$ & $\begin{array}{l}2.580 \\
2.576\end{array}$ & $\begin{array}{l}21-1321 \\
17-303\end{array}$ & $\begin{array}{l}48 \\
48\end{array}$ \\
\hline $\begin{array}{l}\mathrm{Rb}_{2} \mathrm{Zn}\left(\mathrm{SO}_{4}\right)_{2} \cdot 6 \mathrm{H}_{2} \mathrm{O} \\
\mathrm{Rb}_{2} \mathrm{Fe}\left(\mathrm{SO}_{4}\right)_{2} \cdot 6 \mathrm{H}_{2} \mathrm{O}\end{array}$ & $\begin{array}{l}9.185 \\
9.218\end{array}$ & $\begin{array}{l}12.450 \\
12.497\end{array}$ & $\begin{array}{l}6.242 \\
6.256\end{array}$ & $\begin{array}{l}105^{\circ} 55^{\prime} \\
105^{\circ} 45^{\prime}\end{array}$ & $\begin{array}{l}686.42 \\
693.56\end{array}$ & $\begin{array}{l}42 \\
43\end{array}$ & $\begin{array}{l}2.596 \\
2.523\end{array}$ & $\begin{array}{l}21-1328 \\
21-1322\end{array}$ & $\begin{array}{l}48 \\
48\end{array}$ \\
\hline $\begin{array}{l}\mathrm{Cs}_{2} \mathrm{Mg}\left(\mathrm{SO}_{4}\right)_{2} \cdot 6 \mathrm{H}_{2} \mathrm{O} \\
\mathrm{Cs}_{2} \mathrm{Ni}\left(\mathrm{SO}_{4}\right)_{2} \cdot 6 \mathrm{H}_{2} \mathrm{O}\end{array}$ & $\begin{array}{l}9.330 \\
9.264\end{array}$ & $\begin{array}{l}12.848 \\
12.773\end{array}$ & $\begin{array}{l}6.360 \\
6.359\end{array}$ & $\begin{array}{l}107^{\circ} \\
106^{\circ} 59^{\prime}\end{array}$ & $\begin{array}{l}728.97 \\
719.66\end{array}$ & $\begin{array}{l}42 \\
42\end{array}$ & $\begin{array}{l}2.689 \\
2.883\end{array}$ & $\begin{array}{l}20-615 \\
21-163\end{array}$ & $\begin{array}{l}48 \\
48\end{array}$ \\
\hline $\begin{array}{l}\mathrm{Cs}_{2} \mathrm{Cu}\left(\mathrm{SO}_{4}\right)_{2} \cdot 6 \mathrm{H}_{2} \mathrm{O} \\
\mathrm{Cs}_{2} \mathrm{Co}\left(\mathrm{SO}_{4}\right)_{2} \cdot 6 \mathrm{H}_{2} \mathrm{O}\end{array}$ & $\begin{array}{l}9.439 \\
9.316\end{array}$ & $\begin{array}{l}12.762 \\
12.824\end{array}$ & $\begin{array}{l}6.310 \\
6.365\end{array}$ & $\begin{array}{l}106^{\circ} 11^{\prime} \\
107^{\circ} 7^{\prime}\end{array}$ & $\begin{array}{l}729.93 \\
726.74\end{array}$ & $\begin{array}{l}42 \\
14\end{array}$ & $\begin{array}{l}2.864 \\
2.856\end{array}$ & $\begin{array}{l}21-846 \\
17-305\end{array}$ & $\begin{array}{l}48 \\
48\end{array}$ \\
\hline $\begin{array}{l}\mathrm{Cs}_{2} \mathrm{Zn}\left(\mathrm{SO}_{4}\right)_{2} \cdot 6 \mathrm{H}_{2} \mathrm{O} \\
\mathrm{Cs}_{2} \mathrm{Fe}\left(\mathrm{SO}_{4}\right)_{2} \cdot 6 \mathrm{H}_{2} \mathrm{O}\end{array}$ & $\begin{array}{l}9.316 \\
9.355\end{array}$ & $\begin{array}{l}12.815 \\
12.893\end{array}$ & $\begin{array}{l}6.373 \\
6.378\end{array}$ & $\begin{array}{l}106^{\circ} 57^{\prime} \\
106^{\circ} 53^{\prime}\end{array}$ & $\begin{array}{l}727.83 \\
736.16\end{array}$ & $\begin{array}{l}42 \\
42\end{array}$ & $\begin{array}{l}2.881 \\
2.805\end{array}$ & $\begin{array}{l}20-719 \\
20-402\end{array}$ & $\begin{array}{l}48 \\
48\end{array}$ \\
\hline $\mathrm{Cs}_{2} \mathrm{Mn}\left(\mathrm{SO}_{4}\right)_{2} \cdot 6 \mathrm{H}_{2} \mathrm{O}$ & 9.425 & 12.970 & 6.389 & $107^{\circ} 10^{\prime}$ & 746.27 & 42 & 2.763 & $21-847$ & 48 \\
\hline
\end{tabular}

a Powder Data File issued by the Joint Committee on Powder Diffraction Standards. 
TABLE 6. Tutton salts-chromates, monoclinic, $\mathrm{P} 2{ }_{1} / \mathrm{a}$ (No. 14)

\begin{tabular}{|c|c|c|c|c|c|c|c|c|c|}
\hline \multirow{2}{*}{ Compound } & \multicolumn{4}{|c|}{ Cell size } & \multirow{2}{*}{$\begin{array}{l}\text { Vol. } \\
\left(\AA^{3}\right)\end{array}$} & \multirow{2}{*}{$\begin{array}{l}\text { Refer- } \\
\text { ence }\end{array}$} & \multirow{2}{*}{$\begin{array}{l}\text { Density } \\
\mathrm{g} / \mathrm{cm}^{3}\end{array}$} & \multirow{2}{*}{$\begin{array}{l}\mathrm{PDF}^{\text {a }} \\
\text { No. }\end{array}$} & \multirow{2}{*}{$\begin{array}{l}\text { Other } \\
\text { references }\end{array}$} \\
\hline & $a(\AA)$ & $b(\AA)$ & $c(\AA)$ & $\beta$ & & & & & \\
\hline $\begin{array}{l}\left(\mathrm{NH}_{4}\right)_{2} \mathrm{Mg}\left(\mathrm{CrO}_{4}\right)_{2} \cdot 6 \mathrm{H}_{2} \mathrm{O} \\
\mathrm{Rb}_{2} \mathrm{Mg}\left(\mathrm{CrO}_{4}\right)_{2} \cdot 6 \mathrm{H}_{2} \mathrm{O}\end{array}$ & $\begin{array}{l}9.508 \\
9.474\end{array}$ & $\begin{array}{l}12.674 \\
12.571\end{array}$ & $\begin{array}{l}6.246 \\
6.256\end{array}$ & $\begin{array}{l}106^{\circ} 14^{\prime} \\
104^{\circ} 57^{\prime}\end{array}$ & $\begin{array}{l}722.64 \\
719.86\end{array}$ & $\begin{array}{l}43 \\
43\end{array}$ & $\begin{array}{l}1.757 \\
2.470\end{array}$ & $\begin{array}{l}21-701 \\
22-1282\end{array}$ & 2 \\
\hline $\begin{array}{l}\mathrm{Cs}_{2} \mathrm{Mg}\left(\mathrm{CrO}_{4}\right)_{2} \cdot 6 \mathrm{H}_{2} \mathrm{O} \\
\left(\mathrm{NH}_{4}\right)_{2} \mathrm{Ni}\left(\mathrm{CrO}_{4}\right)_{2} \cdot 6 \mathrm{H}_{2} \mathrm{O}\end{array}$ & $\begin{array}{l}9.604 \\
9.420\end{array}$ & $\begin{array}{l}12.952 \\
12.603\end{array}$ & $\begin{array}{l}6.369 \\
6.275\end{array}$ & $\begin{array}{l}106^{\circ} 6^{\prime} \\
105^{\circ} 54^{\prime}\end{array}$ & $\begin{array}{l}761.23 \\
716.45\end{array}$ & $\begin{array}{l}43 \\
43\end{array}$ & $\begin{array}{l}2.750 \\
2.016\end{array}$ & $\begin{array}{l}21-844 \\
21-705\end{array}$ & 2 \\
\hline
\end{tabular}

${ }^{a}$ Powder Data File issued by the Joint Committee on Powder Diffraction Standards.

[15] Hill, A. E., and Yanick, N. S., J. Am. Chem. Soc. 5 7, 645, (1935).

[16] Hofmann, W., Z. Krist. 78, 279 (1931).

[17] Hofmann, W., Z. Krist 82, 323 (1932).

[18] Hofmann, W., Z. Krist. 75, 158 (1930).

[19] Hölemann, H., Z. Anorg. Allgem. Chem. 239, 257 (1938).

[20] Jona, F., and Pepinsky, R., Phy. Rev. 103, 1126 (1956).

[21] Kannan, K. K., and Viswamitra, M. A., Z. Krist. 122, 161 (1965).

[22] Kohler, K., and Franke, W., Mineralogishes Mt., Freise Un., Berlin, Germany, in Powder Data File.

[23] Kohler, K., and Franke, W., Z. Anorg. Allgem. Chem. 331, 27 (1964).

[24] Kohler, K., and Franke, W., Z. Anorg. Allgem. Chem. 331, 17 (1964).

[25] Lepeshkov, I. N., and Noviskova, L. N., Russ. J. Inorg. Chem. (Eng. Trans.) 3, 2395 (1958).

[26] Lindsley, L. C., and Dennis, L. M., J. Am. Chem. Soc. 47, 377 (1925).

[27] Mallet, F. R., J. Chem. Soc. 77, 216 (1900).

[28] Mallet, F. R., J. Chem. Soc. 81, 1546 (1902).

[29] Margulis, T. N., and Templeton, D. H., Z. Krist. 11 7, 344 (1962).

[30] Montgomery, H., Chastain, R. V., and Lingafelter, E. C., Acta Cryst. 20, 731 (1966).

[31] Montgomery, H., Chastain, R. V., Natt, J. J., Witkowska, A. M., and Lingafelter, E. C., Acta Cryst. 22, 775 (1967).

[32] Montgomery, H., and Lingafelter, E. C., Acta Cryst 17, 1478 (1964).

[33] Montgomery, H., and Lingafelter, E. C., Acta Cryst. 17, 1295 (1964).

[34] Montgomery, H., and Lingafelter, E. C., Acta Cryst. 20, 659 (1966).

[35] Montgomery, H., and Lingafelter, E. C., Acta Cryst. 20, 728 (1966).

[36] Morey, G. W., Rowe, J. J., and Fournier, R. O., J. Inorg. Nucl. Chem. 26, 55 (1964).

[37] Mukherjee, P. L., Z. Krist. 91 A, 504 (1935).

[38] Mukherjee, P. L., Indian J. Phys. 18, 148 (1944).

[39] Plyushchev, V. E., Russ. J. Inorg. Chem. (Eng. Trans.) 7, 709 (1962).

[40] Ramsdell, L. S., Am. Mineralogist, 20, 569 (1935).

[41] Swanson, H. E., McMurdie, H. F., Morris, M. C., and Evans, E. H., Nat. Bur. Stand. (U.S.), Monogr. 25, Sec. 6, 101 pages (1968).
[42] Swanson, H. E., McMurdie, H. F., Morris, M. C., and Evans, E. H., Nat. Bur. Stand. (U.S.), Monogr. 25, Sec. 7, 186 pages (1969).

[43] Swanson, H. E., McMurdie, H. F., Morris, M. C., and Evans, E. H., Nat. Bur. Stand. (U.S.), Monogr. 25, Sec. 8 (1970).

[44] Tret'yakov, Yu. D., Russ. J. Inorg. Chem. (Eng. Trans.) 6, 501 (1961).

[45] Tutton, A. E. H., Z. Krist. 33, 1 (1900).

[46] Tutton, A. E. H., Z. Krist. 27, 113 (1897).

[47] Tutton, A. E. H., J. Chem. Soc. 87, 1123 (1905).

[48] Tutton, A. E. H., J. Chem. Soc. 63, 337 (1893).

[49] Tutton, A. E. H., J. Chem. Soc. 69, 344 (1896).

[50] Tutton, A. E. H., Phil. Tran. Roy. Soc. London, Ser. A, 216, 1 (1916).

[51] Tutton, A. E. H., Phil. Tran. Roy. Soc. London, Ser. A, 197, 255 (1901).

[52] Tutton, A. E. H., Phil. Tran. Roy. Soc. London, Ser. A, 21 7, 199 (1919).

[53] Tutton, A. E. H., Proc. Roy. Soc. (London), Ser. A, 118, 367 (1928).

[54] Tutton, A. E. H., Proc. Roy. Soc. (London), Ser. A, 83, 211 (1910).

[55] Tutton, A. E. H., Proc. Roy. Soc. (London), Ser. A, 88, 361 (1913).

[56] Tutton, A. E. H., Proc. Roy. Soc. (London), Ser. A, 108, 240 (1925).

[57] Tutton, A. E. H., Proc. Roy. Soc. (London), Ser. A, 67, 58 (1900).

[58] Tutton, A. E. H., Proc. Roy. Soc. (London), Ser. A, 101, 245 (1922).

[59] Tutton, A. E. H., Proc. Roy. Soc. (London), Ser. A, 101, 225 (1922).

[60] Tutton, A. E. H., Proc. Roy. Soc. (London), Ser. A, 96, 156 (1920).

[61] Tutton, A. E. H., Proc. Roy. Soc. (London), Ser. A, 98, 67 (1921).

[62] Tutton, A. E. H., and Porter, M. W., Mineral. Mag. 16, 169 (1912).

[63] Weidenborner, J. E., Tsu, I., and Godycki, L. E., Acta Cryst. 14, 63 (1961).

[64] Zemann, A., and Zemann, J., Acta Cryst. 10, 409 (1957).

(Paper 75A5-677) 